\title{
Real-time Monitoring of Structural Vibration using Spectral-domain Optical Coherence Tomography
}

\author{
Shuncong Zhong ${ }^{1,2}$, Hao Shen ${ }^{1}$, Yaochun Shen ${ }^{1 *}$ \\ ${ }^{1}$ Department of Electrical Engineering and Electronics, University of Liverpool, \\ Liverpool L69 3GJ, UK \\ ${ }^{2}$ School of Mechanical Engineering and Automation, Fuzhou University, P.R.China
}

\begin{abstract}
: we report in this paper the development of a spectral-domain optical coherence vibration tomography (OCVT) using a broadband CCD-based spectrometer and a shortcoherence white light source. We demonstrate that both the vibration amplitude and frequency can be quantified, in the frequency range of $0-250 \mathrm{~Hz}$, with an axial resolution of $1 \mu \mathrm{m}$. Furthermore, the inner structure (layer thickness) of a vibrating sample can also be quantified simultaneously. The developed OCVT is non-contact and noninvasive in nature, thus is ideal for real time and in situ monitoring of low-frequency micro-vibrations that have critic impacts on many high-precision manufacturing and engineering processes.
\end{abstract}

Keywords: Structural vibration; Optical coherence tomography; Non-destructive testing

\section{Introduction}

In recent years, there has been an increasing demand from industrial and scientific organizations for vibration monitoring and measurement techniques in applications such as high-

\footnotetext{
* Corresponding author. Tel: +44 (0)151 794 4575; fax: +44 (0)151 7944540.

E-mail address: y.c.shen@liv.ac.uk (Dr. Yaochun Shen)
} 
precision manufacturing, and micro electromechanical system (MEMS) [1-3]. Strain gauge, accelerometer, optical fibre sensor have been employed in the vibration measurements [3-6]. However, these methods require direct contact with the sample and this may limit their applications. Recently, vibration and deformation have been successfully characterised using various non-contact optical interferometers techniques [7-12]. Generally, the displacement is obtained from the phase change of the interferometric patterns, providing extremely high depth resolution (down to $10^{-12} \mathrm{~m}$ ). However, the detection range is usually limited to half of the wavelength of the laser source owing to the $2 \pi$ phase ambiguity [11]. The detection range can be extended beyond the half wavelength limit by using phase shift/modulation techniques, at the cost of increased instrument complexity and decreased measurement accuracy (related to the inherent phase shift error) [13-14]. On the other hand, Laser Doppler Vibrometre (LDV) directly measures the velocity of a sample although the displacement information can also be obtained by the integration of velocity along the time axis $[6,15]$.

In all these methods mentioned, only the displacement of a sample is obtained, no information on the inner structure of a sample could be revealed. In this paper, we will demonstrate that by directly measuring the absolute displacement of a sample using optical coherence tomography (OCT), one can obtain both the inner structure (layer thickness) and the vibration parameters (amplitude and frequency) of the sample simultaneously and in real time. 


\section{Experiment}

Fig.1 shows the schematic diagram of a spectral-domain optical coherence vibration tomography (OCVT). The light from a $50 \mathrm{~W}$ halogen lamp is delivered into a Michelson interferometer using a biconvex lens. Light is then split into reference and sample beams by a beam-splitter (50/50). Both the "object image" and the "reference image" are formed at the entrance slit of a broadband CCD-based spectrometer (USB2000+, Ocean Optics, USA). Interference occurs when both the "object image" and the "reference image" are spatially matched in size and orientation, and their optical path lengths are matched within the coherence length of the light source. The sample used here is a plastic substrate coated with a polymer thin film of $\sim 25 \mu \mathrm{m}$ thickness. The sample is attached to a louder speaker that acts as the vibration exciter.

The spectral interferogram of the interferometer can be expressed as

$$
I(\lambda, t)=\sqrt{\frac{\varepsilon_{0}}{\mu_{0}}}\left|E_{r}(\lambda)+E_{s}(\lambda)\right|^{2}
$$

where $\varepsilon_{0}$ and $\mu_{0}$ are the vacuum permittivity and the vacuum permeability, respectively; the electrical filed from reference $\left(E_{r}\right)$ and sample $\left(E_{s}\right)$ can be written as [16]

$$
E_{r}(\lambda)=S_{r} E_{i}(\lambda) \exp (j 2 \pi t c / \lambda)
$$




$$
E_{s}(\lambda)=S_{s}(\lambda) E_{i}(\lambda) \exp (j 2 \pi t c / \lambda-j 2 \pi \Delta l(t) / \lambda)
$$

where $E_{i}(\lambda)$ is the electrical filed of incidence light; $c$ and $\lambda$ is the velocity and the wavelength of light in air; $\Delta l(t)$ is the optical path length difference between the reference and sample arm, which is a function of $t$ for a vibrating sample. $S_{r}$ is the reflection coefficient of a reference (mirror) and is wavelength-independent whilst $S_{s}(\lambda)$ is the reflection coefficient of the sample which is usually wavelength-dependent for a layered sample. For simplicity, we assume (1) the sample has a single coating layer of a thickness of $d$; (2) the contribution from the multiple reflections within the coating is negligible. The reflection coefficient from a layered sample [17] can then be simplified as:

$$
S_{s}(\lambda)=S_{s 0}(\lambda)+S_{s 1}(\lambda) \exp (-j 4 \pi d / \lambda)
$$

where $S_{s 0}(\lambda)$ and $S_{s 1}(\lambda)$ corresponds to the surface reflection and the coating/core interface reflection of the sample, respectively. Using Eqs. (2) - (4), Eq. (1) can be rewritten as:

$$
\begin{aligned}
I(\lambda, t) & =I_{0}(\lambda) S_{r}{ }^{2}+I_{0}(\lambda)\left(S_{s 0}(\lambda)^{2}+S_{s 1}(\lambda)^{2}\right) \\
& +2 I_{0}(\lambda) S_{r} S_{s 0}(\lambda) \cos [2 \pi \Delta l(t) / \lambda] \\
& +2 I_{0}(\lambda) S_{r} S_{s 1}(\lambda) \cos [2 \pi \Delta l(t) / \lambda+4 \pi d / \lambda] \\
& +2 I_{0}(\lambda) S_{s 0} S_{s 1}(\lambda) \cos (4 \pi d / \lambda)
\end{aligned}
$$


where the intensity of the incident light is $I_{0}(\lambda)=\sqrt{\frac{\varepsilon_{0}}{\mu_{0}}}\left|E_{i}(\lambda)\right|^{2}$. The first two terms in this equation are the mean (DC) intensities returning from the reference and sample arms of the interferometer, respectively. The third and fourth terms in this equation, which depends on the optical path length difference $\Delta l(t)$, represents the amplitude of the interference fringes that carry information about the surface position and the inner structures of the sample, respectively. The last term, the auto-correlation term, in the equations represents the interference fringe between the surface and the interface of the sample. Note that for a vibrating sample the optical path length difference is no longer a constant. The Fast Fourier transform (FFT) of the measured spectral interferogram provides both the surface position (i.e. $\Delta L(t)$,) and the depth profile of the sample (i.e., $I_{s}(\lambda, t) \rightarrow I_{s}(z, t)$, where $z$ is the depth direction).

\section{Results and Discussion}

\subsection{Characterization of inner structures of a static sample by OCVT}

The insert of Fig. 1 shows a typical spectral interferogram measured for a static sample. The measured spectrum has a centre wavelength of $\lambda_{0}=700 \mathrm{~nm}$ and a full width at half-maximum (FWHM) of $\Delta \lambda=236 \mathrm{~nm}$. Interference fringes are visible across the whole spectral range of $400-1000 \mathrm{~nm}$. The OCT axial resolution is calculated to be $0.9 \mu \mathrm{m}$ in air, using the expression of $\frac{2 \ln 2}{\pi} \frac{\lambda_{0}^{2}}{\Delta \lambda}[18]$. Fig.2 shows the corresponding OCT-FFT waveform (FFT amplitude of the 
measured spectral interferogram). The main peak at $0 \mu \mathrm{m}$ corresponds to the sample surface and the second peak at $38 \mu \mathrm{m}$ corresponds to the polymer/plastic interface of the sample. The physical thickness of the layer $d_{2}$ is determined to be $25 \mu \mathrm{m}$, assuming that the refractive index $n$ of the layer is 1.5. Generally, refractive index is defined as $n=v / c$, where $c$ and $v$ are the velocity of light in air and in medium, respectively; The physical thickness of the layer $d_{2}$ can be calculated as: $d_{2}=d_{1} / n$, where $d_{1}$ is the corresponding optical path length in air. Note that the axial resolution (defined as the FWHM of the main peak) achieved is $1 \mu \mathrm{m}$ in air, one of the best ever reported. In addition, the second peak at $38 \mu \mathrm{m}$ has a much smaller amplitude of 0.023 , equivalent to about $1 \%$ change in the refractive index at the interface between the polymer coating and the plastic substrate. Therefore the OCVT developed provides a very efficient way for probing minor refractive index changes within a sample.

One distinct feature of our OCVT system is the combination of a broadband CCD-based spectrometer with a white light source [19] which has an extremely short coherence length. This configuration provides not only excellent axial resolution of $1 \mu \mathrm{m}$ in air (sun-micrometer in a sample), but also allows the whole spectral interferogram to be measured in a single-shot (all frequency components were measured simultaneously by the linear sensor array of the spectrometer).

\subsection{Characterization of a sample vibrating at a single-frequency by OCVT}


This single-shot experiment scheme, together with the achieved sub-micrometer axial resolution, makes it possible to use the OCVT for quantitatively characterizing vibrating samples in real time. For this purpose, the same sample was mounted onto a loud speaker whose vibration frequency and amplitude was controlled by a computer. Fig.2 shows the snapshot of the OCTFFT waveform measured at the time of 140, 156 and $190 \mathrm{~ms}$, respectively. As expected, the position of the main peak of the OCT-FFT waveform changes with time, because the sample was vibrating along axial direction. In addition, the extracted coating thickness of the sample remained constant at all three positions, suggesting that there is no measurable deformation of the sample used here.

Fig.3 (a) shows the 150 OCT-FFT waveforms of the vibrating sample recorded continuously over a period of $300 \mathrm{~ms}$. The layer thickness can again be obtained from the peak position difference between the main and the small peaks. Furthermore, the peak position of the first main peak varies in a sinusoidal manner. The insert of Fig. 3 shows the plot of the peak position as a function of time, with Fig. 3 showing its corresponding FFT amplitude. The vibration frequency and the amplitude are subsequently retrieved to be $10 \mathrm{~Hz}$ and $8.1 \mu \mathrm{m}$, respectively.

The OCT-FFT waveform (that corresponds to the interference fringes) can be expressed as $I(z, t)=A(z, t) \exp (-i \phi(z, t))[20]$. In this paper, as demonstrated in Fig.3, both the vibration amplitude and frequency of a vibrating sample are quantified by directly measuring the 
amplitude change $A(z, t)$ between successive OCT scans. The surface displacement of a vibrating sample is obtained in real time by tracking the main peak position of the OCT-FFT waveform (Figure 3). The vibration amplitude of the sample is then calculated as the $\max / \mathrm{min}$ of the surface displacement (inset of Figure 3 (b)). Subsequently, by performing FFT on the temporal vibration signal, the vibration frequency can be quantified as well (Figure 3 (b)). Furthermore, the layer thickness can be obtained from the peak position difference between the main and the small peaks in OCT-FFT waveform of each OCT scan, as shown in Fig.2 and Fig.3. If there is no measurable deformation of the sample, as is the case in this study, the extracted layer thickness of the sample will remain constant for all the OCT scans. Therefore, the OCVT system developed here is a powerful tool for quantifying both the vibration characteristics and the inner structures of a sample simultaneously and in real time. We note that the phase change $\Delta \phi(z, t)$ between successive OCT scan can also be used to characterize the inner structure of a sample, for example, to quantify tissue deformations [20-21]. As an example, Fig. 4(a) shows the 2D maps of another vibrating sample which has a polymer film of $27 \mu \mathrm{m}$ thickness (in air), recorded continuously in a period of $3000 \mathrm{~ms}$. Here, The vibration amplitude and frequency were determined, by analyzing the amplitude $(A(z, t))$ and the phase $(\Delta \phi(z, t))$ of the recorded waveform, to be $1.5 \mu \mathrm{m}$ and $1.5 \mathrm{~Hz}$, respectively. This was done in a similar manner as aforementioned amplitude-change method: by firstly tracking the feature position of each OCT- 
FFT waveform, followed by performing Fourier transform of the resulting vibration curve. It can be seen from Fig. 4 that the resolutions of both the amplitude and phase maps are high and, therefore, they are both suitable for measurement of the low-frequency and ultra-fine-amplitude vibration. In addition, there is an oscillation feature between the surface and interface in the 2D phase map (Fig.4 (b)), whilst not shown on the 2D amplitude map. This indicates that the phase map is more sensitive to the minor inner-structures of a sample, albeit it is also more sensitive to measurement noise. Note that the straight lines at a depth of 5-10 microns correspond to the autocorrelation of the sample. Auto-correction artefact is due to the auto- and cross-correlated terms introduced by the reflections from various interfaces presented within the sample [22]. A phase shifting method has been used in the static OCT to eliminate the auto-correction artefact. In the vibration OCT reported here, the phase shifting method was not used as the auto-correlation is a time-independent "DC" term (refer to Eq. (5)) thus would not affect the vibration measurement.

\subsection{Characterization of a sample vibrating at multi-frequencies by OCVT}

As a further demonstration of the OCVT capability, we drive the loudspeaker with a complex signal which has 4 frequency components (the combination of 10, 70, 160 and $210 \mathrm{~Hz}$ signals, the corresponding amplitude of the voltages added on the loudspeaker are $0.1,0.07,0.07$ and 0.1 volts respectively). Again the spectral interferogram was recorded continuously for a period of $3000 \mathrm{~ms}$ and the extracted surface position was then plotted as a function of time in Fig. 5 (a) 
(for clarity only the first $500 \mathrm{~ms}$ results were shown). It is evident from Fig.5 (b) that all the vibration frequencies of the sample are successfully identified, demonstrating that the OCVT can be used for characterising sample vibrating at multiple frequencies.

In our current OCVT system, the minimum integration time of the spectrometer is limited to 1 ms, corresponding to 1000 measurements per second. This allows us to characterise vibrations with a frequency up to $250 \mathrm{~Hz}$. This could be easily increased to a few $\mathrm{KHz}$ if the latest CCDbased spectrometer is employed. On the other hand, it has been shown that the surface quality of the finished products in high-precision machining is not only related to the amplitude but also to the frequency of the vibrations [1]. The frequencies, which affect the performance of most highprecision engineering, are from $0.5 \mathrm{~Hz}$ to $70 \mathrm{~Hz}$ [1]. Therefore the proposed OCVT system is an ideal tool for in-situ monitoring of these low-frequency micro-vibrations in high-precision manufacturing and measuring system $[1,3]$. The amplitude range of the micro-vibrations using the current vibration OCT system is about $0.1-80 \mu \mathrm{m}$. The maximum measurable amplitude of the micro-vibrations is limited by the spectrometer resolution $\delta \lambda$ and is given by $z_{\max }=\frac{\lambda_{0}^{2}}{4 \delta \lambda}$, where $\delta \lambda$ is the spectral resolution and $\lambda_{0}$ is the centre wavelength of the spectrum. For our current setup, $\lambda_{0}=700 \mathrm{~nm}$ and $\delta \lambda=1.5 \mathrm{~nm}$, and this results a maximum measurable amplitude of about $80 \mu \mathrm{m}$. A spectrometer with higher spectral resolution will improve this significantly. The minimum measurable amplitude of the micro-vibration, on the other hand, is ultimately limited 
by the accuracy of the resolved peak position of the OCT-FFT waveform. In our setup this is better than $0.1 \mu \mathrm{m}$, although Liang et al [20] reported an extremely-high sensitivity at subnanometer level in studying tissue deformation. Spectral-domain OCT [18] was employed which is a non-invasive and cross-sectional imaging technique and which permits, for example, micrometer-resolution three-dimensional images to be obtained from within the retina [17]. Previously it has also been used for detection and characterization of subsurface Hertzian cracks in ceramic materials [23], and for the in vivo thickness measurement of sweat film layer [24]. Liang et al. [20] used OCT technique to quantify the deformation of tissues that are subjected to slow dynamic compression (e.g. at about $0.1 \mathrm{~Hz}$ ). The surface of the tissue was fixed. Our work is the first application of vibration OCT in quantifying both the surface displacement (amplitude and frequency) and the inner structures (layer thickness) of a sample vibrating at frequencies up to $250 \mathrm{~Hz}$.

\section{Conclusions}

In summary, we have introduced a new technique for quantitatively characterizing the vibration and the inner structure a sample simultaneously and in real time. The OCVT system developed can quantitatively characterise vibrations with a frequency up to $250 \mathrm{~Hz}$, with an axial resolution of $1 \mu \mathrm{m}$. The OCVT is non-contact and noninvasive in nature, thus is ideal for real time and in situ monitoring of vibration in high-precision manufacture sector. 


\section{Acknowledgement}

The work was supported by UK Technology Strategy Board (AB293H), Fuzhou University

Research Fund and the Scientific Research Foundation for the Returned Overseas Chinese Scholars, State Education Ministry, P. R. China.

\section{Nomenclature}

$\varepsilon_{0} \quad$ the vacuum permittivity

$\mu_{0} \quad$ the vacuum permeability

$\lambda$ the wavelength of the light

$E_{r}(\lambda) \quad$ the electrical filed from reference

$E_{s}(\lambda) \quad$ the electrical field from sample

$E_{i}(\lambda) \quad$ the electrical filed of incidence light

$S_{r} \quad$ the reflection coefficient of the reference

$S_{s}(\lambda) \quad$ the reflection coefficient of the sample

$I(\lambda, t) \quad$ the spectral interferogram

$t \quad$ time

$\Delta l(t) \quad$ the optical path length difference between the reference and sample arm (for vibrating sample)

d thickness of a single coating of a sample;

$S_{s 0}(\lambda) \quad$ the surface reflection of the sample 


$\begin{array}{ll}S_{s 1}(\lambda) & \text { the coating/core interface reflection of the sample } \\ I_{0}(\lambda) & \text { the intensity of the incident light } \\ z & \text { the depth direction } \\ \lambda_{0} & \text { centre wavelength of the light } \\ \Delta \lambda & \text { a full width at half-maximum (FWHM) } \\ n & \text { velocity of light in air } \\ C & \text { velocity of light in medium } \\ v & \text { the thickness of the layer in air } \\ d_{1} & \text { the physical thickness of the layer } \\ d_{2} & \text { amplitude change between successive OCT scan } \\ A(z, t) & \text { the phase change between successive OCT scan } \\ \Delta \phi(z, t) & \text { the amplitude detection range of the micro-vibrations by using vibration OCT } \\ Z_{\text {max }} & \text { the spectromer resolution }\end{array}$

\section{References}

[1] J.Z. Zhang, D. Li, M.J. Chen and S. Dong. An ultra-low frequency parallel connection nonlinear isolator for precision instruments, Key Engineering Materials. Key Engineering Materials 2004; 257-258; 231-6.

[2] S. Jordan, E. Lawrence. Vibration nullification of MEMS device using input shaping. Proceedings of SPIE Smart Structures 2003.

[3] Y. Nakamura, M. Nakayama, M. Kura, M. Yasuda and T Fujita. Application of active microvibration control system using a giant magnetostrictive actuator. Journal of intelligent Material Systems and Structures 2007; 18: 1137-48. 
[4] R.C. Turner, P.A. Fuierer, R.E. Newnham, and T.R. Shrout. Materials for high temperture acoustic and vibration sensors: a review. Applied Acoustics 1994; 41: 299-324.

[5] S. Zhong, and S. O. Oyadiji, Resopnse-only method for damage detection of beam-like structures using high accuracy frequencies with auxiliary mass spatial probing, Journal of Sound and Vibration 2008; 311: 1075-99

[6] K. Kageyama, I. Kimpara, T. Suzuki, I. Ohsawa, H. Murayama, and K. Ito. Smart marine structures: an approach to the monitoring of ship structures with fibre optic sensors. Smart Materials and Structures 1998; 7: 472-8.

[7] Y.C. Shen, P. Hess. Real-time detection of laser-induced transient gratings and surface acoustic wave pulses with a Michelson interferometer. Journal of Applied Physics 1997; $82: 4758-62$

[8] Z. Li, S. Zhen, B. Chen, M. Li, R. Liu and B. Yu. Lissajous figures in the application of micro-vibration measurement. Optics Communication 2008; 281: 4744-6.

[9] G. He, X. Wang. Real-time micro-vibration measurement in sinusoidal phase-modulating interferometry. Optik 2009; 120: 101-5.

[10] K. Kokkonen, M. Kaivola. Scanning heterodyne laser interferometer for phase-sensitive absolute-amplitude measurements of surface vibrations. Applied Physics Papers 2008; 92: 063502.

[11] R. Dauksevicius, A. bubulis, V. Jurenas, V. Ostasevicius and S. Tamulevicius. Investigation of dynamics of cantilever-type microstructure by laser Doppler vibrometry. Ultragarsas 2004; 3:29-32.

[12] X. Li, G. Tao. Low-frequency harmonic vibration analysis with temporal speckle pattern interferometry. Optics \& Laser Technology 2002; 34: 259-64.

[13] B. Dhanasekar, and B. Ramamoorthy. Digital speckle interferometry for assessment of surface roughness. Optics and Lasers in Engineering 2008; 46: 272-80.

[14] A. Patil, R. Langoju, and P Rastogi. Phase shifting interferometry using a robust parameter 
estimation method . Optics and Lasers in Engineering 2007; 45: 293-7.

[15] R. B. Northrop, Introduction to instrumentation and measurements (2nd), Taylor and Francis.

[16] N. Tan-no, T. Ichimura, T. Funaba, N. Anndo, and Y. Odagiri, Optical multimode frequency-domain reflectometer, Optics Letters 1994; 19(8): 587-9.

[17] Y.C. Shen, P.F. Taday, Development and Application of Terahertz Pulsed Imaging for Nondestructive Inspection of Pharmaceutical Tablet (invited paper), J. Selected Topics in Quantum Electronics 2008; 14 : 407-15.

[18] A.F. Fercher, W. Drexler, C.K. Hitzenberger, and T. Lasser, Optical coherence tomography—principles and applications, Report on Progress in Physics 2003; 66: 239-303.

[19] A.F. Fercher, C.K. Hitzenberger, M. Sticker, E. Moreno-Barriuso, R. Leitgeb, W. Drexler, and H. Sattmann. A thermal light source technique for optical coherence tomography. Optics Communication 2000; 185: 57-64.

[20] R. K. Wang, S. Kirkpatrick, and M. Hinds. Phase-sensitive optical coherence elastography for mapping tissue microstrains in real time. Applied Physics Letters 2007; 90: 164105.

[21] Xing Liang, Amy L. Oldenburg, Vasilica Crecea, EricJ. Chaney, and Stephan A. Boppart. Optical micro-scale mapping of dynamic biomechanical tissue properties. Optics Express 2008; 16(15): 11052-65.

[22] Z. Ma, R. K. Wang, F. Zhang and J. Yao, Spectral optical coherence tomography using twophase shifting method, Chinese Physics Letter 2005; 22(8): 1909-12.

[23] M. Bashkansky, D. Lewis III, V. Pujari, J. Reintjes, and H.Y. Yu. Subsurface detection and characterization of Hertzian cracks in Si3N4 balls using optical coherence tomography. NDT\& E Internatinonal 2001; 34: 547-55.

[24] Enock Jonathan, In vivo sweat film layer thickness measured with Fourier-domain optical coherence tomography (FD-OCT), Optics and Lasers in Engineering 2008; 46(6): 424-7 


\section{Captions:}

Fig.1. Schematic diagram of the spectral-domain OCVT experimental set-up. The sample vibration is excited by a loud speaker. THL, Tungsten Halogen Lamp ; EP, Entrance pupil; L, Lens; REF, Reference; BS, Beam splitter; OF, Optical fiber; S, Samples; LS, Loud speaker. The insert shows a typical spectral interferogram measured using this setup.

Fig.2. The snapshot of OCT-FFT waveform of a micron-vibrating sample measured at the time of $140 \mathrm{~ms}$ (curve A), $156 \mathrm{~ms}$ (curve B) and $190 \mathrm{~ms}$ (curve C), respectively. The dashed doublearrowhead indicates the optical thickness of the polymer thin film $(\sim 38 \mu \mathrm{m}$ in air). The OCTFFT waveform of the same sample at static is shown as the top curve. For clarity, all OCT-FFT waveforms are offset vertically.

Fig.3. (a) 3D map comprising 150 OCT-FFT waveforms of the vibrating sample recorded continuously in a period of $300 \mathrm{~ms}$. The color scale corresponds to signal strength. The main peaks around $z=0 \mu \mathrm{m}$ ( $z$ is depth direction) illustrate the surface position of a vibrating sample whilst the distance between the main and the small peak corresponds to layer thickness. For clarity, the signal above $25 \mu \mathrm{m}$ was enlarged by a factor 5. (b) The frequency distribution of the vibrating sample. The insert show the plot of the peak position as a function of time.

Fig.4. Comparison of the amplitude (a) and phase (b) 2D maps of a sample with a polymer film 
of $27 \mu \mathrm{m}$ thickness (in air), recorded continuously in a period of $3000 \mathrm{~ms}$. The sample is vibrating a frequency of $1.5 \mathrm{~Hz}$ with an amplitude of $1.5 \mu \mathrm{m}$.

Fig.5. The measured surface position of a vibrating sample as a function of time (a), and its corresponding FFT amplitude (b). Vibration frequencies at $10 \mathrm{~Hz}, 70 \mathrm{~Hz}, 160 \mathrm{~Hz}$ and $210 \mathrm{~Hz}$ were identified, which agrees well with the frequencies of the applied excitation signal. 


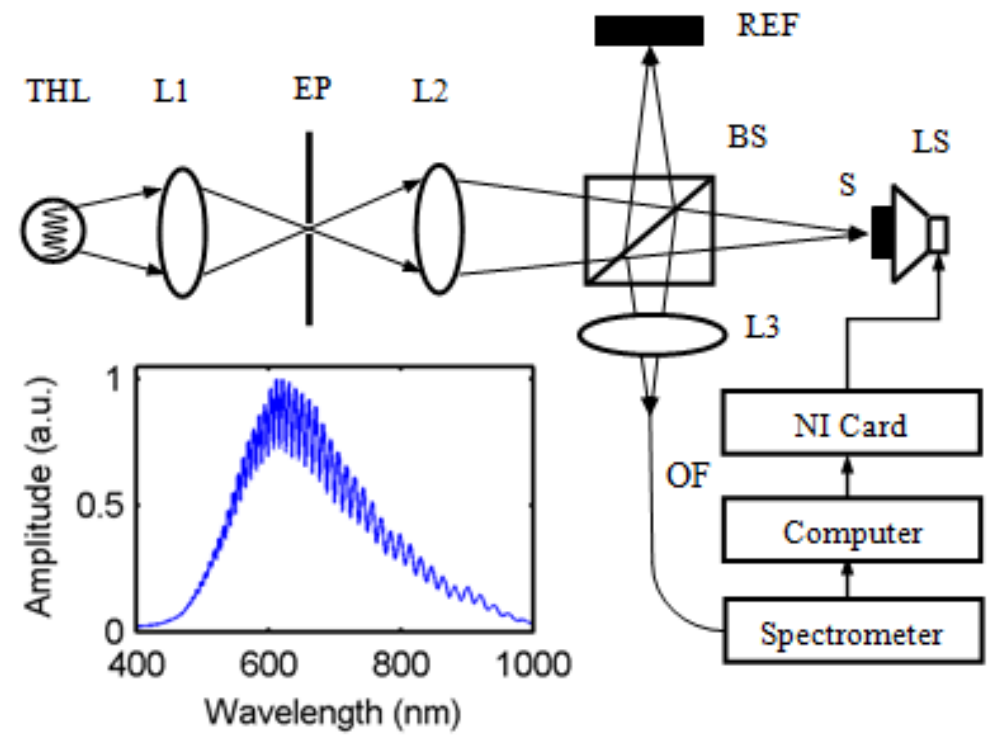

Fig.1

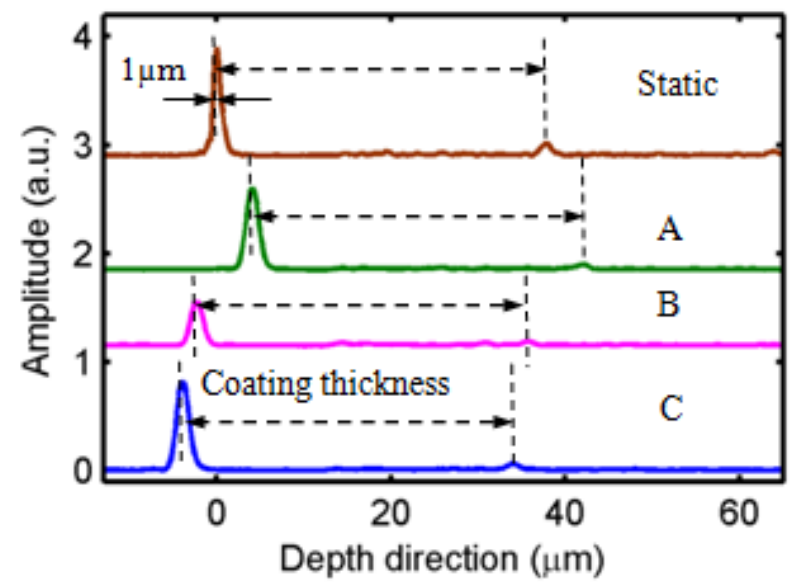

Fig.2 
(a)

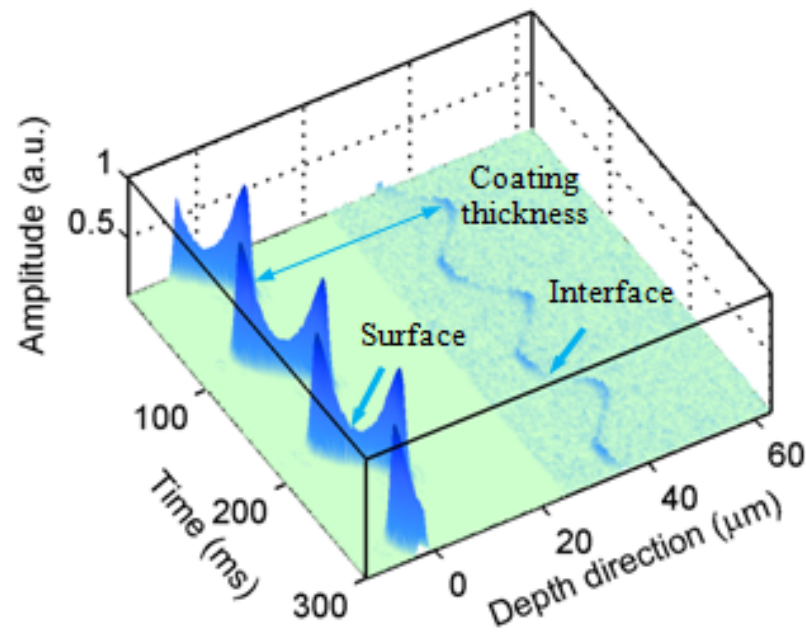

(b)

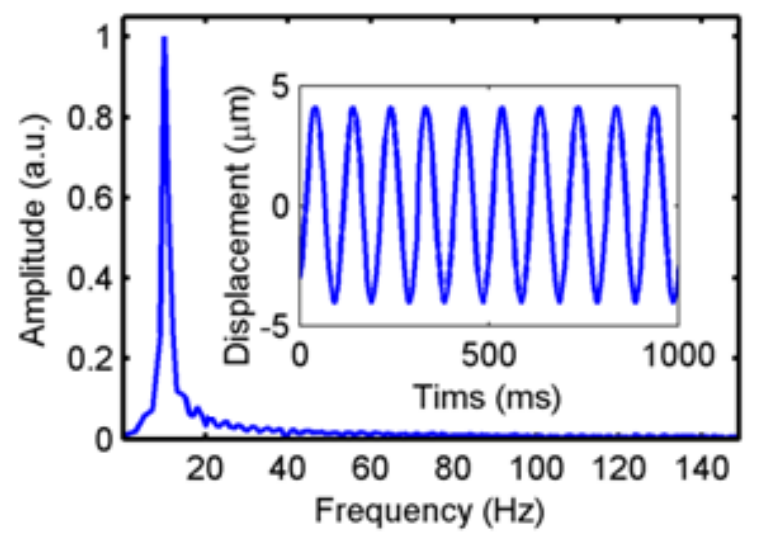

Fig.3 

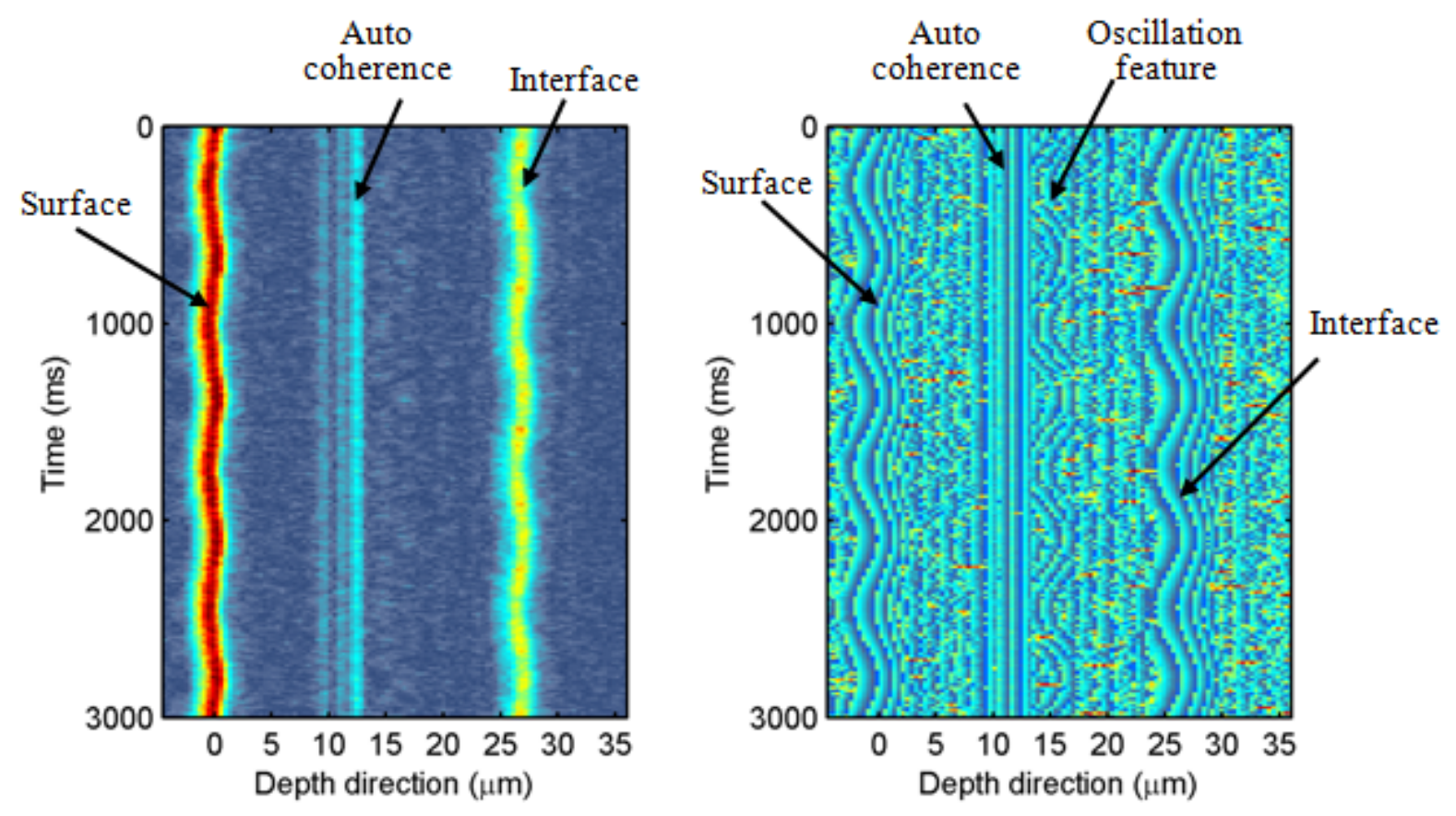

Fig.4

(a)

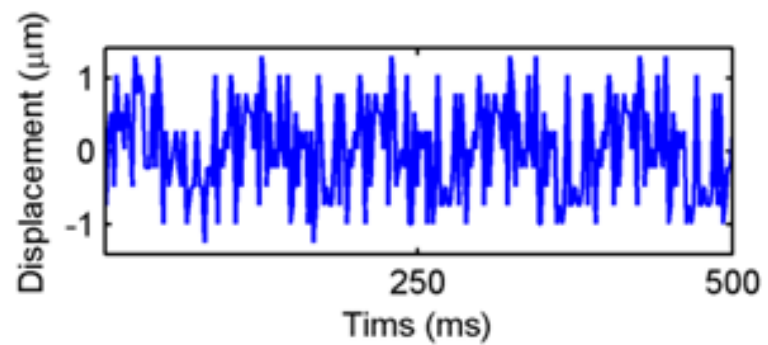

(b)

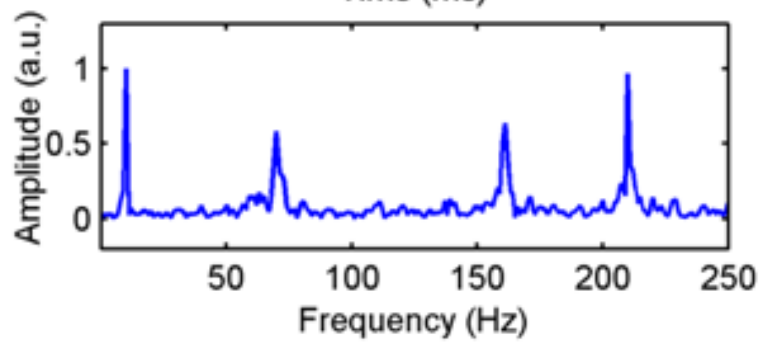

Fig. 5 